\title{
Effects of a Combined Nutraceutical on Lipid Pattern, Glucose Metabolism and Inflammatory Parameters in Moderately Hypercholesterolemic Subjects: A Double-blind, Cross-over, Randomized Clinical Trial
}

\author{
Arrigo Francesco Giuseppe Cicero ${ }^{1,2}$ - Alessandro Colletti ${ }^{1} \cdot$ Federica Fogacci $^{1}$. \\ Marilisa Bove $^{1} \cdot$ Martina Rosticci $^{1} \cdot$ Claudio Borghi $^{1}$
}

Received: 7 May 2016/Accepted: 7 June 2016/Published online: 23 June 2016

(C) The Author(s) 2016. This article is published with open access at Springerlink.com

\begin{abstract}
Background There is an increasing interest for combined nutraceuticals that can act on several points of lipid and glucose metabolism with preventive purposes. However, the simple assemblage of nutraceuticals with potentially additive mechanism of action need to be clinically tested. Methods To assess the effects of a combination of nutraceuticals based on artichoke, red yeast rice, banaba, and coenzyme Q10, we performed a double bind, crossover designed trial versus placebo in 30 adults with LDL cholesterol suboptimal in primary prevention of cardiovascular disease. After a period of 3 weeks of dietary habits correction, patients began a period of 6 weeks of treatment with nutraceutical or placebo, followed by 2 weeks of washout and finally 6 weeks in cross-over. Data related to lipid pattern, insulin resistance, renal function, liver and CPK have been obtained at each visit.

Results In particular, the after the nutraceutical treatment the enrolled patients experienced a significant improvement in total cholesterol $(-13.6 \%)$, LDL-C $(-18.2 \%)$, non-HDL-C $(-15 \%)$, glutamic oxaloacetic transaminase $(-10 \%)$, glutamate-pyruvate transaminase $(-30.9 \%)$, and hs-CRP $(-18.2 \%)$ versus placebo. No changes have been observed in the other investigated parameters in both groups.

Conclusions The tested combination of nutraceuticals has shown clinical efficacy in the reduction of total cholesterol,
\end{abstract}

Arrigo Francesco Giuseppe Cicero

arrigo.cicero@unibo.it

1 Department of Medicine and Surgery Sciences, University of Bologna, Bologna, Italy

2 Hypertension Research Center, Poliambulatorio Pad. 2, Via Albertoni, 15, 40138 Bologna, Italy
non-HDL, LDL and triglycerides, while improving the level of liver transaminases and high sensitivity C-reactive protein. Further confirmation are needed to verify these observations on the middle and long term with a larger number of subjects.

Keywords Nutraceuticals · Monacolin K · Insulinsensitivity $\cdot$ Dyslipidemia $\cdot$ Artichoke $\cdot$ Banaba $\cdot$ Coenzyme Q10

\section{Introduction}

Cardiovascular disease is the leading cause of death in the developed countries. The main risk factors, evaluated by the INTERHEART case-control study, are dyslipidemia, smoking, psychosocial factors, diabetes and hypertension [1]. In particular, dyslipidemia is considered the principle modifiable risk factor, because of its role in the development of atherosclerosis and clinical events [2]. Guidelines for dyslipidemia management suggest a treatment that include lifestyle modification and pharmacotherapy based on identification of groups considered at high, medium or low risk of major cardiovascular events [3]. Nevertheless, lifestyle changes are not always sufficient to reach desirable cholesterol levels, in particular in high-risk subjects, but also in low risk patients that have sub-optimal lipid parameters.

Nutraceuticals are borderline devices between nutrients and drugs providing a supplementation of particular nutrients with beneficial effects on health. More than 40 nutraceutical substances have been studied in order to improve lipid metabolism and some clinical trials demonstrated a cholesterol lowering activity, an improvement of glucose metabolism and inflammatory parameters, besides 
a possible positive influence on cardiovascular prognosis [4]. Among them, red yeast rice, artichoke, banaba and coenzyme Q10 showed interesting results.

Red yeast rice is a nutraceutical produced by fermenting white rice with the yeast, Monascus purpureus. Its content in monacolins is responsible of its lipid-lowering properties, by the inhibition of hydroxymethylglutaryl-coenzyme A reductase, the rate limiting step in cholesterol synthesis. Many clinical trials have found red yeast rice effective at improving the lipid profile. It has also been used as an alternative to drugs in patients with mild to moderate hypercholesterolemia and in subjects intolerant to statin therapy due to statin-associated myalgia. Furthermore, a large randomized controlled study significantly showed that the intake of red yeast rice improve the risk of major adverse cardiovascular events and overall survival in patients following myocardial infarction [5].

The artichoke (Cynara scolymus) inhibits cholesterol biosynthesis [6] and of low-density lipoprotein (LDL) oxidation, improving endothelial function [7]. Moreover, clinical studies have shown also choleretic, hepatoprotective [8] and glucose lowering activity [9].

Lagerstroemia speciosa, commonly known as banaba, is a tropical plant found in many parts of South-East Asia. In the Philippines, the dried and shredded banaba leaves are used as a treatment for diabetes and kidney disease. Many studies in vivo and in vitro confirmed the antidiabetic activity of banaba, due to both corosolic acid as well as ellagitannins [10]. The mechanisms of action involved include enhanced cellular uptake of glucose, impaired hydrolysis of sucrose and starches, decreased gluconeogenesis and the regulation of lipid metabolism. These activities can be mediated by peroxisome proliferator-activated receptor (PPAR), mitogenactivated protein kinase (MAP K), NF- $\kappa \mathrm{B}$ and other signal transduction factors. The supplementation with banaba has demonstrated to be safe and well tolerated in clinical trials [11]. Moreover, its action on both lipid and glucose metabolism make banaba a potential useful tool also in the management of metabolic syndrome [12].

Coenzyme Q10 (CoQ10) is a naturally occurring component present in living cells. The potential use of coenzyme Q10 supplements may help prevent or treat some pathologies, in particular cardiovascular diseases, high blood pressure, obesity and diabetes. It acts as an essential cofactor for ATP production and to perform important antioxidant activities in the body [13]. Moreover, it inhibits LDL oxidation and thus the progression of atherosclerosis. It decreases pro-inflammatory cytokines and decreases blood viscosity, which could be useful in subjects with heart failure and coronary artery disease [14]. This is important because low-grade inflammation and oxidative stress are the key factors in the pathogenesis and development of atherosclerosis and its complications [15].
The association of more nutraceuticals has demonstrated a synergistic effects, in particular in patients that present two or more risk factors for cardiovascular diseases $[16,17]$.

This clinical trial has the aim to test the effectiveness and safety of a combination of red yeast rice, artichoke, banaba and coenzyme Q10 on glucose metabolism, lipid pattern and inflammatory parameters in hypercholesterolemic patients.

\section{Materials and Methods}

\subsection{Study Design}

This double blind, placebo-controlled, randomized clinical trial was carried out in 40 moderately hypercholesterolemic patients with metabolic syndrome, in primary prevention for cardiovascular diseases, consecutively enrolled in the ambulatory service of cardiovascular disease prevention in the Medical and Surgical Sciences Department of the University of Bologna.

The study was a cross-over designed trial of 18 weeks, preceded by 3 weeks of diet stabilization in which patients received standard behavioural and qualitative (not quantitative) dietary suggestions to correct unhealthy habits. In particular, subjects were instructed to follow a general indication of a Mediterranean diet, avoiding excessive intake of dairy products and red meat derived products during the study, and maintaining overall constant dietary habits. Individuals were also generically encouraged to increase their physical activity by walking briskly for 20-30 min, 3-5 times per week, or by cycling.

Inclusion criteria were:

- age between 25 and 75

- LDL-cholesterol (LDL-C) level between 130 and $190 \mathrm{mg} / \mathrm{dL}$

- Body mass index (BMI) value between 25 and $30 \mathrm{~kg} / \mathrm{m}^{2}$

- Triglycerides (TG) level between 150 and $400 \mathrm{mg} / \mathrm{dL}$

- Fasting plasma glucose (FPG) level under $125 \mathrm{mg} / \mathrm{dL}$.

- When present, assumption of lipid-lowering or antihypertensive drugs stabilized since at least 6 months.

Exclusion criteria were:

- secondary prevention for cardiovascular diseases

- assumption of antidiabetic drugs

- inconstant or not-stabilized assumption of drugs which may interfere with lipid or glucose metabolism

- chronic gastrointestinal diseases and assumption of drugs for their treatment

- known thyroid, liver, renal or muscle diseases 
- known allergy or intolerance to a component of the tested product

- any medical or surgical condition which could lead to an inconstant adhesion to the protocol.

The study was fully conducted in accordance with the Declaration of Helsinki, its protocol was approved by the Ethical Committee of the University of Bologna, and informed consent was obtained from all patients before the inclusion in the study.

\subsection{Treatments}

After 4 weeks of diet and physical activity stabilization, patients were allocated to treatment with an indistinguishable pill of placebo or with an active product containing $500 \mathrm{mg}$ of artichoke extract, $200 \mathrm{mg}$ of red yeast rice (corresponding to $10 \mathrm{mg}$ of monacolin $\mathrm{K}$ ), $75 \mathrm{mg}$ of banaba extract, $50 \mathrm{mg}$ of coenzyme Q10, $9 \mathrm{mg}$ of vitamin B3, $1.4 \mathrm{mg}$ of vitamin B6, $0.83 \mathrm{mcg}$ of vitamin B12, $110 \mathrm{mcg}$ of folic acid, kindly offered by Named SpA (Lesmo, Italy).

After 6 weeks of treatment (or placebo), patients were asked to observe a 2-week washout period, and they were then assigned to the alternative treatment for a further period of 6 weeks. Clinical and laboratory data have been obtained at the baseline, at the end of the first treatment period, after the washout, and again after the second treatment period.

Randomization was done assigning an alphabetical code to each lot code (corresponding to treatment or placebo) impressed on the pillbox. Codes were then kept in a sealed envelope, which was not opened until the end of the trial. Pillboxes were then mixed, and the investigators assigned a blinded pillbox to each enrolled patient.

Throughout the study, we instructed patients to take the product first dose on the day after they were given the study product. At the same time, all unused products were retrieved to the investigator.

\subsection{Assessments}

Personal data, CVD history and pharmaceutical anamnesis of each patient were inquired at the beginning of the trial. Anthropometrical parameters, such as body weight, waist circumference and BMI were collected both at the beginning and during the trial, together with hemodynamic and biochemical parameters. BMI was calculated as weight in kilograms divided by the square of height in meters. Hemodynamic parameters were collected measuring orthostatic and clinostatic systolic and diastolic blood pressure (mean value of 3 interval measures). Also orthostatic and clinostatic wrist blood pressure (mean value of 3 interval measures) and cardiac frequency were collected.
All biochemical parameters were obtained after a $12-\mathrm{h}$ overnight fast. The following parameters were obtained or calculated through appropriate formulas: total cholesterol (TC), high-density lipoprotein-cholesterol (HDL-C), TG, LDL-C, non-HDL cholesterol (non-HDL-C), FPG, fasting insulin, homeostatic model assessment of insulin resistance (HOMA-IR), creatinine, serum uric acid (SUA), liver transaminases [glutamic oxaloacetic transaminase (GOT); glutamate-pyruvate transaminase (GPT)], gamma glutamyl-transferase $(\gamma \mathrm{GT})$, and hsCRP [18]. All measurements were centrally performed in the laboratory of our department with standardized methods [19].

HOMA-IR is the simplest and most commonly used method to estimate insulin resistance. It was calculated as the product of basal glucose $(\mathrm{mmol} / \mathrm{L})$ and fasting insulin (U/mL) divided by 22.5 [20].

Adherence to behavioural counselling and to treatment/placebo, tolerability, acceptability and compliance were also assessed.

\subsection{Statistical Analyses}

Data have been analysed using intention to treat by mean of the Statistical Package for Social Science (SPSS) 21.0, version for Windows. Normally distributed baseline characteristics of the population have been compared using Student's $t$ test, non-normally distributed parameters using Mann-Whitney U test. Two-way analyses of variance for crossover design were used to assess the effect of treatment during the assumption of placebo or treatment. All data are expressed as means and standard deviation (SD). To verify the basic assumptions of crossover design, besides the evaluation of period effect, the presence of a carryover effect was excluded. Level of statistical significance was set on $P=0.05$.

\section{Results}

The main characteristics of the enrolled patients have been resumed in Table 1.

At the baseline, the two treatment sequence groups were balanced for all the investigated parameters. All the patients completed the study and no one experienced adverse events during the trial.

Compared to baseline values, no change has been observed after both treatments as it regards Body weight, body mass index, waist circumference, diastolic blood pressure (DBP), pulse pressure (PP), heart rate (HR), creatinine and SUA level (Table 2).

On the other hand, significant improvements have been reported in lipid, hepatic and inflammatory parameters in the active group versus placebo. In particular, there have 
Table 1 Baseline characteristics of the subjects enrolled in the trial

\begin{tabular}{|c|c|c|}
\hline N. 30 & Mean & SD \\
\hline Age (years) & 52.20 & 7.0 \\
\hline Weight (kg) & 80.6 & 7.5 \\
\hline Body mass index $\left(\mathrm{kg} / \mathrm{m}^{2}\right)$ & 26.7 & 1.8 \\
\hline Waist circumference $(\mathrm{cm})$ & 99.8 & 7.6 \\
\hline $\mathrm{SBP}(\mathrm{mmHg})$ & 134.2 & 8.6 \\
\hline DBP (mmHg) & 80.4 & 3.4 \\
\hline Pulse pressure $(\mathrm{mmHg})$ & 54.1 & 6.3 \\
\hline MAP (mmHg) & 97.5 & 7.5 \\
\hline Heart rate (bpm) & 72.4 & 4.4 \\
\hline FPG (mg/dL) & 106.9 & 4.9 \\
\hline Insulin $(\mu \mathrm{U} / \mathrm{mL})$ & 12.5 & 3.3 \\
\hline HOMA-IR & 3.4 & 0.8 \\
\hline Total cholesterol $(\mathrm{mg} / \mathrm{dL})$ & 248.7 & 14.3 \\
\hline LDL-C (mg/dL) & 165.2 & 13.2 \\
\hline Triglycerides (mg/dL) & 219.4 & 17.1 \\
\hline HDL-C (mg/dL) & 40.1 & 2.9 \\
\hline Non HDL-C (mg/dL) & 205.8 & 13.4 \\
\hline GOT (U/L) & 22.7 & 6.7 \\
\hline GPT (U/L) & 21.9 & 6.3 \\
\hline Serum uric acid $(\mathrm{mg} / \mathrm{dL})$ & 4.3 & 0.9 \\
\hline Creatinine $(\mathrm{mg} / \mathrm{dL})$ & 0.9 & 0.1 \\
\hline hsCRP (mg/dL) & 2.2 & 0.4 \\
\hline
\end{tabular}

SBP systolic blood pressure, $D B P$ diastolic blood pressure, $M A P$ mean arterial pressure, $L D L-C$ LDL cholesterol, $H D L-C$ HDL cholesterol, non $H D L-C$ non HDL cholesterol, $F P G$ fasting plasma glucose, HOMA-IR homeostatic model assessment of insulin resistance, GOT glutamic oxaloacetic transaminase, GPT glutamatepyruvate transaminase, $h s C R P$ high sensitivity $\mathrm{C}$-reactive protein

been a mean reduction of total cholesterol of $-34.1 \mathrm{mg} / \mathrm{dL}$ $(-56.8 ;-11.3 \mathrm{mg} / \mathrm{dL} ; \mathrm{P}<0.001)$, LDL-cholesterol of $-30.3 \mathrm{mg} / \mathrm{dL} \quad(-49.7 ; \quad-7.4 ; \quad \mathrm{P}<0.001), \quad$ non-HDL cholesterol of $-31.2 \mathrm{mg} / \mathrm{dL}(-45.5 ;-8.1 ; \mathrm{P}<0.001)$, GOT of $-2.3 \mathrm{U} / \mathrm{L}(-5.7 ;-0.9 ; \mathrm{P}<0.024)$, GPT of $-6.8 \mathrm{U} / \mathrm{L}(-10.4 ;-1.4 ; \mathrm{P}<0.011)$, hs-CRP of -0.4 $(-0.9 ;-0.1 ; \mathrm{P}<0.019)$.

Fasting plasma glucose and insulin levels also mildly improved in the nutraceutical group, but the observed changes did not reach the statistical significance.

\section{Discussion}

The increase of the average age and the quality of life has caused a raise of cardiovascular diseases in the developed countries, that cannot be contained only with an improvement of lifestyle and the use of drugs, either in terms of costs either of the management of potential side effects
[21]. In fact, even though the improvement in its treatment, cardiovascular diseases remain the first cause of death in the developed countries and it is increasingly necessary a widespread prevention [22]. So, it is important to treat its principle risk factors, since they reach borderline values. Considering that patients must be divided into low, medium and high cardiovascular risk, the primary approach for the first category consists in a change of dietary habits and physical activity [23]. Nevertheless, the improvement of lifestyle is often not enough to get the parameters in the optimal range, while, in these patients, the use of drugs can be excessive and give avoidable side effects. The use of nutraceuticals has demonstrated to be a useful tool, because of its safety, high tolerability and the opportunity to treat more risk factors with only one poly-pill.

In literature, banaba, red yeast rice, artichoke and coenzyme Q10 have been tested mainly alone [24-27] or in association towards only one risk factor. For example, artichoke leaf extract and red yeast rice have been studied in their synergic effect on lipid metabolism [28].

In this clinical trial, the lipid-lowering properties of red yeast rice, the hypoglicemic effects of banaba together with the choleretic, hepatoprotective and bile-enhancing effects of artichoke and the antioxidant activity of coenzyme Q10 have been studied together and have demonstrated to be synergic. The results in fact have shown their effectiveness on lipid metabolism, hepatic and inflammatory parameters and the improvement of glucose metabolism in hypercholesterolemic patients. In particular, the after the nutraceutical treatment the enrolled patients experienced a significant improvement in TC $(-13.6 \%)$, LDL-C $(-18.2 \%)$, non-HDL-C $(-15 \%)$, GOT $(-10 \%)$, GPT $(-30.9 \%)$, and hs-CRP $(-18.2 \%)$. The results are in line with what expected from previous trials carried out on the individual components [29,30], but achieved with a single administration per day of more nutraceuticals combined in a tablet. Of particular interest, is the improvement of liver transaminases, both because usually the increase of transaminases is a common feature with lipid-lowering therapy [31], and because high transaminases could be a marker of liver steatosis, that seems to be per se an emerging risk factor for cardiovascular disease [32].

Nevertheless, our trial has some limitation. The first one is the relatively small sample size, partially balanced by the cross-over design of the trial. The second one is the relatively short duration of the trial, however comparable with the most part of explorative trials carried out with metabolically active nutraceuticals. Then, we have not prescribed a specific diet based on bromatological data, but we gave only general dietary suggestion finalized at avoiding dietary excess in order to simulate more strictly a condition of general practice. 
Table 2 Mean difference in changes of the studied parameters after placebo or nutraceutical treatment $(* \mathrm{P}<0.05$ nutraceutical vs. placebo)

\begin{tabular}{|c|c|c|c|c|c|c|c|c|}
\hline \multirow[t]{3}{*}{ N. 30} & \multicolumn{4}{|c|}{ After nutraceutical treatment } & \multicolumn{4}{|c|}{ After placebo treatment } \\
\hline & \multirow{2}{*}{$\begin{array}{l}\text { Mean difference } \\
\text { in changes }\end{array}$} & \multicolumn{2}{|c|}{$95 \% \mathrm{CI}$} & \multirow[t]{2}{*}{$\mathrm{P}$} & \multirow{2}{*}{$\begin{array}{l}\text { Mean difference } \\
\text { in changes }\end{array}$} & \multicolumn{2}{|c|}{$95 \% \mathrm{CI}$} & \multirow[t]{2}{*}{$\mathrm{P}$} \\
\hline & & Lower & Upper & & & Lower & Upper & \\
\hline$\Delta$ Weight (kg) & -0.2 & -0.7 & 1.0 & 0.621 & -0.3 & -0.9 & 0.8 & 0.798 \\
\hline$\Delta \mathrm{BMI}\left(\mathrm{kg} / \mathrm{m}^{2}\right)$ & -0.1 & -0.6 & 0.9 & 0.712 & -0.1 & -0.4 & 0.7 & 0.684 \\
\hline$\Delta \mathrm{WC}(\mathrm{cm})$ & -0.5 & -1.0 & 0.8 & 0.498 & -0.3 & -1.1 & 0.8 & 0.437 \\
\hline$\Delta \mathrm{SBP}(\mathrm{mmHg})$ & -1.7 & -3.7 & 1.2 & 0.291 & -1.2 & -3.4 & 1.1 & 0.358 \\
\hline$\triangle \mathrm{DBP}(\mathrm{mmHg})$ & -0.9 & -2.6 & 0.3 & 0.092 & -0.6 & -2.1 & 0.5 & 0.275 \\
\hline$\Delta \mathrm{PP}(\mathrm{mmHg})$ & -0.8 & -1.9 & 0.2 & 0.084 & -0.6 & -1.3 & 0.7 & 0.139 \\
\hline$\triangle \mathrm{MAP}(\mathrm{mmHg})$ & -1.2 & -2.2 & 0.9 & 0.192 & -0.9 & -1.6 & 0.6 & 0.121 \\
\hline$\Delta \mathrm{HR}(\mathrm{bpm})$ & 1.4 & -2.3 & 6.6 & 0.544 & -2.1 & -2.9 & 3.4 & 0.484 \\
\hline$\Delta \mathrm{TC}(\mathrm{mg} / \mathrm{dL})$ & -34.1 & -56.8 & -11.3 & $<0.001^{*}$ & -10.1 & -18.3 & 5.6 & 0.060 \\
\hline$\Delta \mathrm{LDL}-\mathrm{C}(\mathrm{mg} / \mathrm{dL})$ & -30.3 & -49.7 & -7.4 & $<0.001 *$ & -8.4 & -16.5 & 4.8 & 0.064 \\
\hline$\Delta$ Triglycerides $(\mathrm{mg} / \mathrm{dL})$ & -19.8 & -44.8 & -9.2 & $<0.001 *$ & -11.4 & -25.6 & 2.5 & 0.051 \\
\hline$\Delta \mathrm{HDL}-\mathrm{C}(\mathrm{mg} / \mathrm{dL})$ & 0.4 & -0.6 & 1.3 & 0.084 & 0.3 & -0.9 & 0.5 & 0.349 \\
\hline$\Delta$ Non HDL-C (mg/dL) & -31.2 & -45.5 & -8.1 & $<0.001^{*}$ & -9.5 & -17.2 & 5.0 & 0.062 \\
\hline$\Delta \mathrm{FPG}(\mathrm{mg} / \mathrm{dL})$ & -5.1 & -9.4 & 4.6 & 0.239 & -3.9 & -8.7 & 6.6 & 0.486 \\
\hline$\Delta$ Insulin $(\mu \mathrm{U} / \mathrm{mL})$ & -1.2 & -1.9 & 0.1 & 0.068 & -0.5 & -1.7 & 0.8 & 0.537 \\
\hline$\Delta$ HOMA-IR & -0.5 & -0.7 & 0.2 & 0.091 & -0.1 & -0.6 & 0.4 & 0.632 \\
\hline$\Delta \mathrm{GOT}(\mathrm{U} / \mathrm{L})$ & -2.3 & -5.7 & -0.9 & $0.024 *$ & 0.3 & -5.9 & 8.5 & 0.734 \\
\hline$\Delta \mathrm{GPT}(\mathrm{U} / \mathrm{L})$ & -6.8 & -10.4 & -1.4 & $0.011^{*}$ & 2.9 & -4.1 & 7.6 & 0.533 \\
\hline$\Delta$ SUA $(\mathrm{mg} / \mathrm{dL})$ & -1.4 & -2.7 & 1.2 & 0.432 & -0.4 & -2.9 & 2.4 & 0.883 \\
\hline$\Delta$ Creatinine $(\mathrm{mg} / \mathrm{dL})$ & -0.2 & -0.5 & 0.8 & 0.547 & 0.2 & -0.3 & 0.5 & 0.638 \\
\hline$\Delta \mathrm{hsCRP}$ & -0.4 & -0.9 & -0.1 & $0.019 *$ & 0.1 & -0.3 & 0.6 & 0.348 \\
\hline
\end{tabular}

$B M I$ body mass index, $D B P$ diastolic blood pressure, $F P G$ fasting plasma glucose, $G O T$ glutamic oxaloacetic transaminase, $G P T$ glutamatepyruvate transaminase, $H D L-C$ HDL cholesterol, HOMA-IR homeostatic model assessment of insulin resistance, $H R$ heart rate, $h S C R P$ high sensitivity C-reactive protein, $L D L-C$ LDL cholesterol, non $H D L-C$ non $\mathrm{HDL}$ cholesterol, $M A P$ mean arterial pressure, $P P$ pulse pressure, $S B P$ systolic blood pressure, SUA serum uric acid, TC total cholesterol, $W C$ waist circumference

\section{Conclusions}

In our clinical trial, the use of combined nutraceuticals has demonstrated to be well tolerated in hypercholesterolemic patients and has shown to be effective in the simultaneous improvement of more risk factors: dyslipidaemia, liver transaminases and hsCRP. These results have to be confirmed in larger and longer trials.

Acknowledgments This work has been carried out with institutional funding from the University of Bologna, Italy.

\section{Compliance with Ethical Standards}

Conflict of interest The authors declare that they have no conflict of interest.

Research involving human participants and/or animals This article does not contain any studies with human participants or animals performed by any of the authors.

Informed Consent For this study informed consent is not required.
Open Access This article is distributed under the terms of the Creative Commons Attribution-NonCommercial 4.0 International License (http://creativecommons.org/licenses/by-nc/4.0/), which permits any noncommercial use, distribution, and reproduction in any medium, provided you give appropriate credit to the original author(s) and the source, provide a link to the Creative Commons license, and indicate if changes were made.

\section{References}

1. Yusuf S, Hawken S, Ounpuu S, Dans T, Avezum A, Lanas F, McQueen M, Budaj A, Pais P, Varigos J, et al. Effect of potentially modifiable risk factors associated with myocardial infarction in 52 countries (the INTERHEART study): case-control study. Lancet. 2004;364:937-52.

2. Hendrani AD, Adesiyun T, Quispe R, Jones SR, Stone NJ, Blumenthal RS, Martin SS. Dyslipidemia management in primary prevention of cardiovascular disease: Current guidelines and strategies. World J Cardiol. 2016;8(2):201-10.

3. Sabatine MS. Advances in the treatment of dyslipidemia. Cleve Clin J Med. 2016;83(3):181-6.

4. Mannarino MR, Ministrini S, Pirro M. Nutraceuticals for the treatment of hypercholesterolemia. Eur J Intern Med. 2014;25(7):592-9. 
5. Burke FM. Red yeast rice for the treatment of dyslipidemia. Curr Atheroscler Rep. 2015;17(4):495.

6. Wider B, Pittler MH, Thompson-Coon J, Ernst E. Artichoke leaf extract for treating hypercholesterolaemia. Cochrane Database Syst Rev. 2013;3:CD003335.

7. Lupattelli G, Marchesi S, Lombardini R, Roscini AR, Trinca F, Gemelli F, Vaudo G, Mannarino E. Artichoke juice improves endothelial function in hyperlipemia. Life Sci. 2004;76(7):775-82.

8. Ben Salem M, Affes H, Ksouda K, Dhouibi R, Sahnoun Z, Hammami S, Zeghal KM. Pharmacological studies of Artichoke leaf extract and their health benefits. Plant Foods Hum Nutr. 2015;70(4):441-53.

9. Rondanelli M, Monteferrario F, Perna S, Faliva MA, Opizzi A. Health-promoting properties of artichoke in preventing cardiovascular disease by its lipidic and glycemic-reducing action. Monaldi Arch Chest Dis. 2013;80(1):17-26.

10. Klein G, Kim J, Himmeldirk K, Cao Y, Chen X. Antidiabetes and Anti-obesity Activity of Lagerstroemia speciosa. Evid Based Complement Alternat Med. 2007;4(4):401-7.

11. Stohs SJ, Miller H, Kaats GR. A review of the efficacy and safety of banaba (Lagerstroemia speciosa L.) and corosolic acid. Phytother Res. 2012;26(3):317-24.

12. Cicero AF, Colletti A. Role of phytochemicals in the management of metabolic syndrome. Phytomedicine. 2015 Dec 11. doi:10.1016/j.phymed.2015.11.009.

13. Garrido-Maraver J, Cordero MD, Oropesa-Avila M, Vega AF, de la Mata M, Pavon AD, Alcocer-Gomez E, Calero CP, Paz MV, Alanis M, de Lavera I, Cotan D, Sanchez-Alcazar JA. Clinical applications of coenzyme Q10. Front Biosci. 2014;19:619-33.

14. Kumar A, Kaur H, Devi P, Mohan V. Role of coenzyme Q10 (CoQ10) in cardiac disease, hypertension and Meniere-like syndrome. Pharmacol Ther. 2009;124(3):259-68.

15. Sarter B. Coenzyme Q10 and cardiovascular disease: a review. J Cardiovasc Nurs. 2002;16(4):9-20.

16. Cicero AF, Colletti A. Combinations of phytomedicines with different lipid lowering activity for dyslipidemia management: The available clinical data. Phytomedicine. 2015 Nov 10. doi:10. 1016/j.phymed.2015.10.011.

17. Volpe R, Sotis G. Nutraceuticals: definition and epidemiological rationale for their use in clinical practice. High Blood Press Cardiovasc Prev. 2015;22(3):199-201.

18. Cicero AF, Morbini M, Rosticci M, D'Addato S, Grandi E, Borghi C. Middle-term dietary supplementation with red yeast rice plus coenzyme Q10 improves lipid pattern, endothelial reactivity and arterial stiffness in moderately hypercholesterolemic subjects. Ann Nutr Metab. 2016;68(3):213-9.

19. Cicero AF, Rosticci M, Reggi A, Derosa G, Parini A, Grandi E, D'Addato S, Borghi C. Relationship between serum uric acid and electrocardiographic alterations in a large sample of general population: data from the Brisighella Heart Study. High Blood Press Cardiovasc Prev. 2015;22(2):129-34.
20. Matthews DR, Hosker JP, Rudenski AS, Naylor BA, Treacher DF, Turner RC. Homeostasis model assessment: insulin resistance and beta-cell function from fasting plasma glucose and insulin concentrations in man. Diabetologia. 1985;28:412-9.

21. Borghi C, Cicero AF. Nutraceuticals with clinically detectable blood pressure lowering effect: a review of available randomized clinical trials and their meta-analyses. $\mathrm{Br} \mathrm{J}$ Clin Pharmacol. 2016;. doi:10.1111/bcp.12902.

22. Banegas JR, López-García E, Dallongeville J, Guallar E, Halcox JP, Borghi C, Massó-González EL, Jiménez FJ, Perk J, Steg PG, De Backer G, Rodríguez-Artalejo F. Achievement of treatment goals for primary prevention of cardiovascular disease in clinical practice across Europe: the EURIKA study. Eur Heart J. 2011;32:2143-52.

23. Saha S, Gerdtham UG, Johansson P. Economic evaluation of lifestyle interventions for preventing diabetes and cardiovascular diseases. Int J Environ Res Public Health. 2010;7:3150-95.

24. Miura T, Takagi S, Ishida T. Management of diabetes and its complications with banaba (Lagerstroemia speciosa L.) and Corosolic Acid. Evid Based Complement Alternat Med. 2012;2012:871495.

25. Cicero AF, Derosa G, Parini A, Maffioli P, D’Addato S, Reggi A, Giovannini M, Borghi C. Red yeast rice improves lipid pattern, high-sensitivity C-reactive protein, and vascular remodeling parameters in moderately hypercholesterolemic Italian subjects. Nutr Res. 2013;33(8):622-8.

26. Rondanelli M, Giacosa A, Opizzi A, Faliva MA, Sala P, Perna S, Riva A, Morazzoni P, Bombardelli E. Beneficial effects of artichoke leaf extract supplementation on increasing HDL-cholesterol in subjects with primary mild hypercholesterolaemia: a double-blind, randomized, placebo-controlled trial. Int J Food Sci Nutr. 2013;64(1):7-15.

27. Hidaka T, Fujii K, Funahashi I, Fukutomi N, Hosoe K. Safety assessment of coenzyme Q10 (CoQ10). BioFactors. 2008;32(1-4):199-208.

28. Barrat E, Zaïr Y, Sirvent P, Chauveau P, Maudet C, Housez B, Derbord E, Lescuyer JF, Bard JM, Cazaubiel M, Peltier SL. Effect on LDL-cholesterol of a large dose of a dietary supplement with plant extracts in subjects with untreated moderate hypercholesterolaemia: a randomised, double-blind, placebo-controlled study. Eur J Nutr. 2013;52(8):1843-52.

29. Cicero AF, Tartagni E, Ertek S. Nutraceuticals for metabolic syndrome management: from laboratory to benchside. Curr Vasc Pharmacol. 2014;12(4):565-71.

30. Cicero AF, Ferroni A, Ertek S. Tolerability and safety of commonly used dietary supplements and nutraceuticals with lipidlowering effects. Expert Opin Drug Saf. 2012;11(5):753-66.

31. Desai CS, Martin SS, Blumenthal RS. Non-cardiovascular effects associated with statins. BMJ. 2014;349:g3743.

32. Francque SM, van der Graaff D, Kwanten WJ. Non-alcoholic fatty liver disease and cardiovascular risk: pathophysiological mechanisms and implications. J Hepatol. 2016 Apr 15. doi:10. 1016/j.jhep.2016.04.005. 